\title{
Divulgación de aspectos ambientales en los informes de sostenibilidad frente al nuevo escenario internacional
}

\section{Disclosure of environmental aspects in sustainability reports in the face of the new international scenario}

\author{
Juan Pablo del Barco Zilli' ${ }^{1}$, María de los Angeles del Barco Zilli², \\ María Sol del Barco Zilli ${ }^{3}$, Ileana García Federici ${ }^{4}$
}

\begin{abstract}
RESUMEN
El cambio climático y las políticas que el mismo propicia pueden traer aparejadas grandes consecuencias para las empresas en particular y para la economía en general. En este contexto donde los riesgos ambientales inciden en los mercados, la importancia de una adecuada presentación de la información no financiera sobre los factores medioambientales se torna inmediata. Una efectiva apertura de información relevante para los inversores requiere una evaluación cuidadosa de los riesgos comerciales prospectivos derivados del cambio climático y de la huella de carbono, entre otros.

Teniendo en cuenta la precariedad y / o ausencia de normas o modelos estandarizados que homogenicen la divulgación y exposición de información en materia de RSE lleva a analizar la información que las organizaciones reportan en la actualidad. En la primera parte del trabajo el objetivo es analizar cuantas de las empresas que cotizan en la bolsa argentina presentan reportes de sostenibilidad y de las que presentan estudiar como reportan los aspectos vinculados con el medio ambiente. En particular se tratará de determinar si hay homogeneidad o no en la información que presentan. En una segunda etapa del trabajo se estudiará la transición de las guías GRI G4 a los nuevos GRI standards, tomando como caso de estudio el Banco Galicia SA que fue la primera empresa argentina en implementarlos.

Se presenta una investigación descriptiva cualitativa, realizada aplicando la técnica de análisis de contenido para los últimos reportes de sostenibilidad publicados por las entidades cotizantes al 2018. Los resultados obtenidos permiten observar que existe dispersión en la exposición de la información, siendo la mayoría de los indicadores de medio ambiente reportados solo por aproximadamente el $50 \%$ de las organizaciones y en lo que respecta a la aplicación de los nuevos estándares no se observan grandes cambios respecto de la última guía GRI.
\end{abstract}

Palabras claves: GRI Standards, Medio Ambiente, Reportes de sostenibilidad, Responsabilidad Social.

Recepción: 13/06/2018. Aprobación: 06/12/2018.

\footnotetext{
1 Universidad Nacional del Litoral, Facultad de Ciencias Económicas, Santa Fe, Argentina, jpdelbarco@fce.unl.edu.ar 2 Universidad Nacional del Litoral, Facultad de Ciencias Económicas, Santa Fe, Argentina, madelbarco@fce.unl.edu.ar 3 Universidad Nacional del Litoral, Facultad de Ciencias Económicas, Santa Fe, Argentina, mdelbarco@fce.unl.edu.ar 4 Universidad Nacional de Rosario, Facultad de Ciencias Económicas y Estadísticas, Rosario, Argentina, igfederici@fcecon.unr.edu.ar
} 


\begin{abstract}
Climate change and the policies it promotes can bring great consequences for organizations in particular and to the economy in general. In this context where markets are affected by environmental risks, the importance of an adequate presentation of non-financial information on environmental factors becomes immediate. An effective disclosure of relevant information for investors requires a careful evaluation of prospective commercial risks derived from climate change and carbon footprint, among others.

The precariousness and/or absence of policies or standardized models that homogenize the disclosure and exposure of CSR information leads to analyze the information currently reported by organizations. The objective of the first part of this paper is to analyze how many listed argentine companies present sustainability reports and among them study how they report on environmental factors. In particular the aim is to determine whether there is or not homogeneity on the information they present. The second part of this paper approaches the transition from G4 GRI guide to the new GRI - STANDARDS, using as case study Banco Galicia SA since it was the first argentine company to implement them.

A qualitative descriptive research was followed applying the content analysis technique to the last sustainability reports published by companies listed at 2018. From the results obtained it can be observed a dispersion on the information disclosure, being the majority of the environmental indicators reported by only approximately $50 \%$ of the organizations, and as regards the application of the new standards it is not observed greater changes with respect to the last GRI guide.
\end{abstract}

Keywords: Environment, GRI Standards, Social Responsibility, Sustainability reports

\title{
INTRODUCCIÓN
}

El cambio climático y las políticas que el mismo propicia pueden traer aparejadas grandes consecuencias para las empresas en particular y para la economía en general. Los riesgos climáticos amenazan la forma en que se desarrolla la vida actual y tienen la capacidad de revertir el avance que se había obtenido en materia de desarrollo y reducción de pobreza, al mismo tiempo que puede generar conflictos producto de las migraciones originadas en la destrucción de los ecosistemas. Las próximas décadas serán decisivas para lograr una profunda transformación de la economía mundial, poniendo el foco en la manera en que los bienes y servicios son producidos, distribuidos y consumidos. Ello así por cuanto la economía mundial sigue siendo carbón, gas y petróleo dependiente, cuando las emisiones de carbono fósiles deben ser reducidas a cero si se pretende que la temperatura global se estabilice (Zenghelis, 2016).

En diciembre de 2015 en la 21 ${ }^{\circ}$ sesión de la Conferencia de las Partes de la Convención Marco de Naciones Unidas sobre el Cambio Climático se celebró el Acuerdo de Paris por el cual la mayoría de los países del mundo, entre ellos Argentina, se comprometen a reducir las emisiones de Gases de Efecto Invernadero en el mediano plazo y aceptan llegar en el largo plazo a la completa descarbonización.

La transición hacia una economía libre de carbono ya está modificando el mercado internacio- 
nal porque a la par que demanda elevados niveles de inversión y propicia innovaciones tecnológicas en energías renovables, desalienta la inversión en activos altos en carbono (por ejemplo en empresas mineras).

En este contexto donde los riesgos ambientales inciden en los mercados, la importancia de una adecuada presentación de la información no financiera sobre los factores medioambientales se torna inmediata (Larrinaga et al., 2002). Una efectiva apertura de información relevante para los inversores requiere una evaluación cuidadosa de los riesgos comerciales prospectivos derivados del cambio climático y de la huella de carbono, entre otros, en otras palabras, los usuarios de la información buscan conocer si las organizaciones han fijado e implementado un proceso efectivo de administración de riesgos, identificando indicadores claves de riesgo y performance (Kerste et al., 2011).

En este sentido el Grupo de Trabajo del Consejo de Estabilidad Financiera sobre Divulgaciones Financieras Relacionadas con el Clima (FSB- TCFD por sus siglas en inglés) propone que "Un acceso más eficaz a los datos mejorará la forma en que se evalúan, tasan y administran, los riesgos relacionados con el clima, de modo que las empresas pueden medir y evaluar con mayor competencia sus propios riesgos y los de sus proveedores y competidores. Los inversores tomarán decisiones mejor informadas, sobre dónde y cómo asignar su capital. Los prestamistas, los aseguradores y los suscriptores podrán evaluar mejor sus riesgos y exposiciones a corto, mediano y largo plazo"

En materia contable, la información presentada por las organizaciones en cuanto a las rendiciones de cuentas se ha ampliado a aspectos no sólo de desempeño económico, sino también social y ambiental, satisfaciendo las necesidades y exigencias de diversos grupos de interés (Larrinaga et al., 2002; Garcia Fronti, 2012). A partir del desarrollo de la contabilidad social y ambiental surgen diversos reportes o informes, llamados reportes de sostenibilidad o balances sociales que complementan la información económica-financiera presentada por la contabilidad patrimonial y financiera tradicional (Moneva, 2005a; Pahlen Acuña y Fronti de Garcia, 2004).

Actualmente es cada vez más importante la información referida a estos aspectos sociales y ambientales, esto unido a la precariedad y/o ausencia de normas o modelos estandarizados que homogenicen su divulgación y exposición lleva a analizar la información que las organizaciones reportan en la actualidad (Hauque et al., 2016).

El presente trabajo se divide en dos partes, en la primera parte el objetivo es analizar cuantas de las empresas que cotizan en la bolsa argentina informan reportes de sostenibilidad y de las que lo realizan estudiar como reportan los aspectos vinculados con el medio ambiente. En particular se tratará de determinar si hay homogeneidad o no en la información que presentan.

En una segunda etapa del trabajo se estudiará la transición de las guías GRI G4 a los nuevos GRI standards, tomando como caso de estudio el Banco Galicia SA siendo la primera empresa argentina en implementarlos.

5 https: / / www.fsb-tcfd.org/about/ 


\section{METODOLOGÍA, MATERIALES Y METODOS}

La presente investigación se configura como descriptiva cualitativa en base al análisis del contenido de los reportes / memorias de sostenibilidad (MS) de las entidades argentinas incluídas por la Comisión Nacional de Valores (CNV) como entidades emisoras al mes de abril 2018.

En esta investigación de tipo descriptiva transversal se emplea la técnica cualitativa de análisis de contenido, la cual permite formular a partir de la información relevada, "inferencias reproducibles y válidas que puedan aplicarse a su contexto" (Krippendorff, 1990, p.28). Esta técnica es la más aplicada en este tipo de investigaciones sobre reportes de sostenibilidad (Gray et al., 1995), teniendo como objetivo conocer el grado de información que presentan las organizaciones en cuanto al aspecto no financiero (Guthrie et al., 2004).

De las 217 entidades incluidas en la CNV a la fecha de corte, se consultaron las páginas web institucionales y la base de datos del Global Reporting Iniciative (GRI) analizando cuáles de las organizaciones han emitido Informes o Reportes de Sostenibilidad. El resultado de esta búsqueda determinó que sólo 47 organizaciones presentaron al menos un informe en materia de RSE, el resto nunca lo hizo o bien solo informa actividades aisladas en su página institucional.

Sobre estas 47 organizaciones que presentan informes de sostenibilidad, se consideró para el análisis de contenido (Krippendorff, 1990) sólo la última memoria presentada bajo la guía G4, obteniendo de esta forma una muestra de 36 reportes. Este análisis de contenido permite determinar las características de las memorias, los indicadores de RSE informados y las tendencias.

En esta investigación se analizaron únicamente los indicadores del aspecto Medio Ambiente, para lo cual se obtuvieron la información de las MS de las entidades, luego se agruparon y se analizaron en conjunto.

Para finalizar la investigación teniendo en cuenta la aplicación a partir de julio del 2018 de los estándares GRI en reemplazo de la guía G4, se realizó el análisis de la información presentada sobre Medio Ambiente por la primera organización que elaboró su informe de RSE aplicando los nuevos estándares (informe 2016), en forma comparativa con el informe anterior (2015) elaborado bajo la guía G4 y con el último informe disponible a la fecha (2017).

\section{RESULTADOS}

Se procede a exponer los resultados en los cuales se informa en primera instancia los datos generales de los reportes de sostenibilidad de las entidades que conforman la muestra y seguidamente los indicadores de la categoría Medio Ambiente reportados por dichas entidades con la información más relevante que se obtuvo del análisis.

De las 217 organizaciones que conforman la población, 47 elaboran memorias de sostenibilidad (MS) y 170 no presentan reportes al momento de la realización del relevamiento. En el gráfico 1 se puede observar el sector económico al que pertenecen las entidades con reportes en materia de RSE. 
Gráfico 1. Clasificación según sector económico de las organizaciones que presentan reportes RSE.

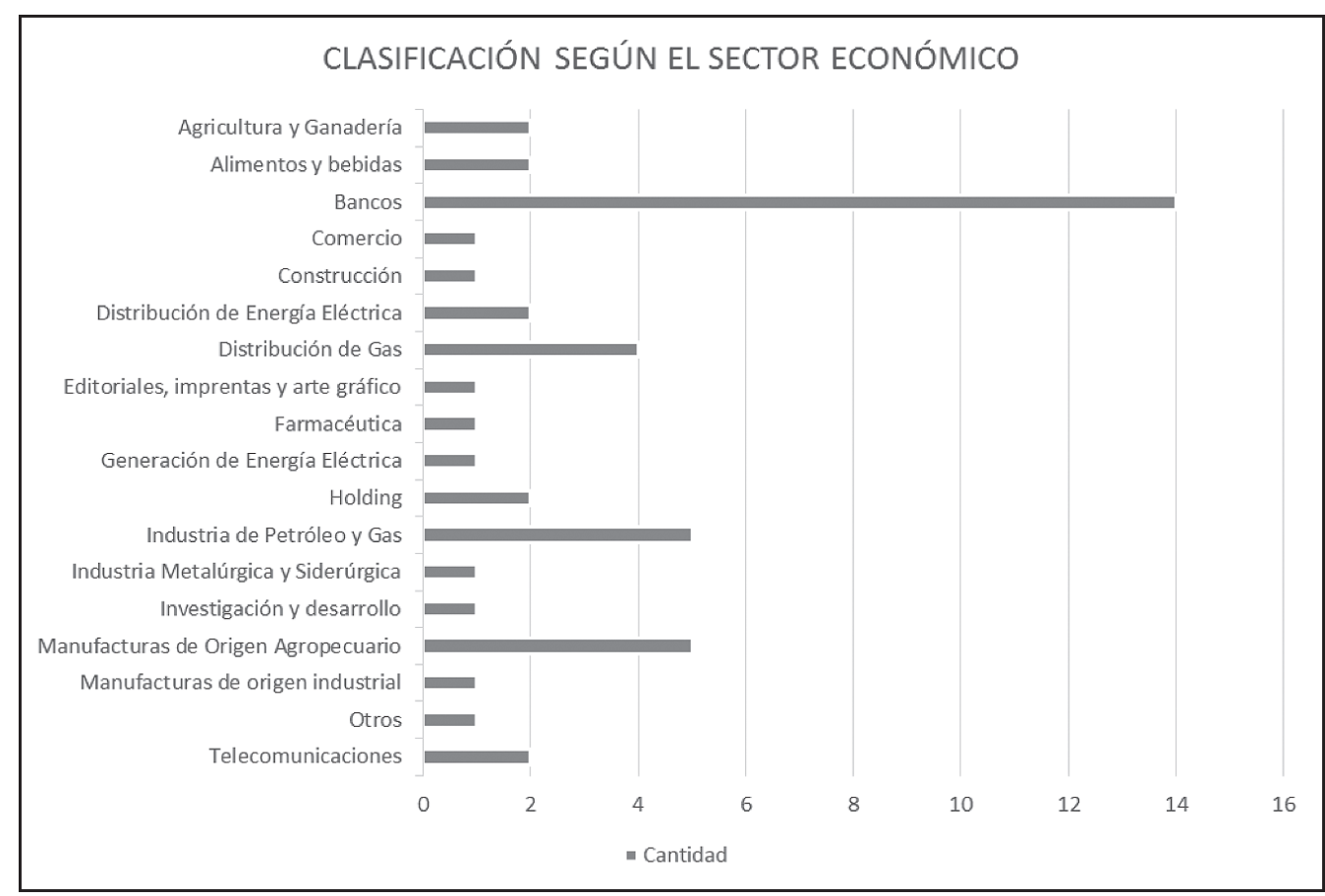

Fuente: Elaboración propia en base a datos de las MS.

En cuanto a la denominación utilizada para los informes de responsabilidad social relevados, se observa en la Tabla 1 la variedad en la definición empleada para estos reportes.

Tabla 1: Denominación utilizada por las organizaciones para la información sobre sostenibilidad.

\begin{tabular}{|l|c|}
\hline \multicolumn{1}{|c|}{ Denominación } & $\begin{array}{c}\text { Organizaciones que presentan } \\
\text { (\%) }\end{array}$ \\
\hline Reporte / Informe / Balance de Sustentabilidad & 57 \\
\hline Memoria / Reporte / Informe de Sostenibilidad & 25 \\
\hline Memoria / Reporte / Informe de RSE & 8 \\
\hline Informe anual Responsabilidad Corporativa & 4 \\
\hline Memoria anual Reporte Integrado & 2 \\
\hline Annual Report and Financial Stantements & 2 \\
\hline Global Report & 2 \\
\hline
\end{tabular}

Fuente: Elaboración propia en base a datos de las MS.

Con respecto al último informe de sostenibilidad presentado por las organizaciones y disponible tanto en sitio web de la entidad o desde la web GRI, en la Tabla 2 se expone el último año reportado y la guía empleada en su elaboración. 
Tabla 2: Último reporte y guía utilizada.

\begin{tabular}{|c|c|c|}
\hline Organizaciones & Último año reportado & Guía utilizada \\
\hline 5 & 2013 & $100 \%$ G3 \\
\hline \multirow{3}{*}{6} & \multirow{3}{*}{2015} & $33 \% \mathrm{G} 3$ \\
\hline & & 17\% G4 Exhaustiva \\
\hline & & $50 \%$ G4 Esencial \\
\hline \multirow{4}{*}{32} & \multirow{4}{*}{2016} & $3 \% \mathrm{G} 3$ \\
\hline & & $6 \%$ No utiliza guía \\
\hline & & 19\% G4 Exhaustiva \\
\hline & & $72 \%$ G4 Esencial \\
\hline \multirow{3}{*}{4} & \multirow{3}{*}{2017} & 25\% G4 Exhaustiva \\
\hline & & $25 \%$ No utiliza guía \\
\hline & & $50 \%$ G4 Esencial \\
\hline
\end{tabular}

Fuente: Elaboración propia en base a datos de las MS.

Es importante destacar, tal como figura en la Tabla 2, que de los 47 entes, ocho realizaron el último informe disponible bajo la guía GRI G3, tres no aplican ninguna guía o estándar y el resto elabora el reporte bajo la última versión G4 (78\% con nivel de aplicación esencial y el 22\% exhaustiva). Sólo 22 entes informan suplemento sectorial que corresponde a los siguientes sectores: $4 \%$ construcción, $14 \%$ energía, $9 \%$ medios, $18 \%$ petróleo y gas, $14 \%$ productos alimenticios, $41 \%$ servicios financieros.

En cuanto al aseguramiento o verificación externa, el cual permite dar credibilidad y legitimidad a los reportes por las actuaciones de empresa ante los grupos de interés (Moneva, 2005b), del relevamiento realizado, solo 14 cuentan con una validación externa, efectuadas en este caso por las grandes firmas de auditoría (7 Deloitte \& Co SA, 2 EY Ernst \& Young, 2 KPMG, 2 PwC y 1 Crowe Horwath).

\section{Análisis del aspecto Medio Ambiente de los reportes}

Para el análisis del aspecto medio ambiente, se acotó la muestra a las organizaciones con memorias de sostenibilidad realizadas bajo la última guía GRI (G4), obteniendo de esta forma 36 organizaciones que informan bajo esta guía.

Como podemos observar en la tabla 3, el indicador más reportado por las organizaciones analizadas es el que refiere a consumo energético interno (EN3) informado por el 81\%, seguido por el indicador de reducción de consumo energético con el $72 \%$ de presentación. Por el contrario los indicadores menos informados son aquellos que reflejan información sobre el porcentaje de los productos vendidos y sus materiales de embalaje que se recuperan al final de su vida útil (EN28 - $81 \%$ ) y de emisiones de sustancias que agotan el ozono (EN20 - 78\%). 
Tabla 3: Indicadores de la Categoría Medio Ambiente de la Guía GRI G4 informados por las organizaciones.

\begin{tabular}{|c|c|c|c|c|}
\hline Aspectos & & Indicador & Reportado & No \\
\hline Energía & EN3 & Consumo energético interno & $81 \%$ & $19 \%$ \\
\hline Energía & EN6 & Reducción del consumo energético & $72 \%$ & $28 \%$ \\
\hline Materiales & EN1 & Materiales por peso o volumen (papel) & $69 \%$ & $31 \%$ \\
\hline Efluentes y Residuos & EN23 & $\begin{array}{l}\text { Peso total de los residuos, según tipo y mé- } \\
\text { todo de tratamiento }\end{array}$ & $67 \%$ & $33 \%$ \\
\hline Agua & EN8 & Captación total de agua según la fuente & $58 \%$ & $42 \%$ \\
\hline Emisiones & EN15 & $\begin{array}{l}\text { Emisiones directas de gases de efecto in- } \\
\text { vernadero }\end{array}$ & $58 \%$ & $42 \%$ \\
\hline $\begin{array}{l}\text { Evaluación ambien- } \\
\text { tal de los provee- } \\
\text { dores }\end{array}$ & EN32 & $\begin{array}{l}\% \text { de proveedores que se examinaron en } \\
\text { función de criterios amb. }\end{array}$ & $58 \%$ & $42 \%$ \\
\hline Efluentes y Residuos & EN27 & $\begin{array}{l}\text { Mitigación del impacto ambiental de los } \\
\text { prod. y servicios }\end{array}$ & $56 \%$ & $44 \%$ \\
\hline Efluentes y Residuos & EN24 & $\begin{array}{l}\text { Número y volumen totales de los derrames } \\
\text { significativos }\end{array}$ & $53 \%$ & $47 \%$ \\
\hline Materiales & EN2 & $\begin{array}{l}\% \text { de los materiales utilizados que son mat. } \\
\text { reciclados (papel) }\end{array}$ & $50 \%$ & $50 \%$ \\
\hline General & EN31 & $\begin{array}{l}\text { Desglose de los gastos y las inversiones } \\
\text { ambientales }\end{array}$ & $50 \%$ & $50 \%$ \\
\hline Efluentes y Residuos & EN22 & $\begin{array}{l}\text { Vertido total de aguas, según su calidad y } \\
\text { destino }\end{array}$ & $47 \%$ & $53 \%$ \\
\hline $\begin{array}{l}\text { Cumplimiento regu- } \\
\text { latorio }\end{array}$ & EN29 & $\begin{array}{l}\text { Valor monetario de las multas y } \mathrm{n}^{\circ} \text { sancio- } \\
\text { nes no monetarias por incumplimiento de } \\
\text { la legislación y la normativa ambiental }\end{array}$ & $47 \%$ & $53 \%$ \\
\hline Emisiones & EN16 & $\begin{array}{l}\text { Emisiones ind.de gases de efecto inv. al ge- } \\
\text { nerar energía }\end{array}$ & $44 \%$ & $56 \%$ \\
\hline Emisiones & EN19 & $\begin{array}{l}\text { Reducción de las emisiones de gases de } \\
\text { efecto invernadero }\end{array}$ & $44 \%$ & $56 \%$ \\
\hline Agua & EN10 & $\begin{array}{l}\text { Porcentaje y volumen total de agua recicla- } \\
\text { da y reutilizada }\end{array}$ & $42 \%$ & $58 \%$ \\
\hline Efluentes y Residuos & EN25 & $\begin{array}{l}\text { Peso de los residuos transportados, impor- } \\
\text { tados, exportados o tratados que se consi- } \\
\text { deran peligrosos }\end{array}$ & $42 \%$ & $58 \%$ \\
\hline $\begin{array}{l}\text { Reclamación am- } \\
\text { biental }\end{array}$ & EN34 & $\begin{array}{l}\text { Número de reclamaciones ambientales que } \\
\text { se han presentado, abordado y resuelto } \\
\text { mediante mecanismos formales de recla- } \\
\text { mación }\end{array}$ & $42 \%$ & $58 \%$ \\
\hline Energía & EN5 & Intensidad energética (por empleado) & $39 \%$ & $61 \%$ \\
\hline Agua & EN9 & $\begin{array}{l}\text { Fuentes de agua que han sido afectadas por } \\
\text { cap.agua }\end{array}$ & $39 \%$ & $61 \%$ \\
\hline Energía & EN7 & $\begin{array}{l}\text { Reducciones de los requisitos energ. de los } \\
\text { prod. y servicios }\end{array}$ & $36 \%$ & $64 \%$ \\
\hline Biodiversidad & EN11 & $\begin{array}{l}\text { Instalaciones operativas que sean adyacen- } \\
\text { tes, contengan o estén ubicadas en áreas } \\
\text { protegidas y áreas no protegidas. }\end{array}$ & $33 \%$ & $67 \%$ \\
\hline
\end{tabular}




\begin{tabular}{|c|c|c|c|c|}
\hline Biodiversidad & EN12 & $\begin{array}{l}\text { Descripción de los impactos en la biodiver- } \\
\text { sidad de áreas protegidas o áreas de alta } \\
\text { biodiversidad no protegidas, derivados de } \\
\text { las actividades, los productos y los servi- } \\
\text { cios }\end{array}$ & $33 \%$ & $67 \%$ \\
\hline Emisiones & EN18 & $\begin{array}{l}\text { Intensidad de las emisiones de gases de } \\
\text { efecto invernadero }\end{array}$ & $33 \%$ & $67 \%$ \\
\hline Emisiones & EN21 & $\begin{array}{l}\text { Nox, sox y otras emisiones atmosféricas } \\
\text { significativa }\end{array}$ & $33 \%$ & $67 \%$ \\
\hline Transporte & EN30 & $\begin{array}{l}\text { Impactos ambientales significativos del } \\
\text { transporte de prod. y otros bienes y mate- } \\
\text { riales utilizados }\end{array}$ & $33 \%$ & $67 \%$ \\
\hline Efluentes y Residuos & EN26 & $\begin{array}{l}\text { Masas de agua y los hábitats relacionados } \\
\text { afectados significativamente por vertidos y } \\
\text { escorrentia procedentes de la org. }\end{array}$ & $31 \%$ & $69 \%$ \\
\hline Energía & EN4 & Consumo energético externo & $28 \%$ & $72 \%$ \\
\hline Emisiones & EN17 & $\begin{array}{l}\text { Otras emisiones indirectas de gases de } \\
\text { efecto invernadero }\end{array}$ & $28 \%$ & $72 \%$ \\
\hline Biodiversidad & EN13 & Hábitats protegidos o restaurados & $25 \%$ & $75 \%$ \\
\hline Biodiversidad & EN14 & $\begin{array}{l}\text { Número de especies cuyos hábitats se en- } \\
\text { cuentran en áreas afectadas por las opera- } \\
\text { ciones. }\end{array}$ & $25 \%$ & $75 \%$ \\
\hline $\begin{array}{l}\text { Evaluación ambien- } \\
\text { tal de los provee- } \\
\text { dores }\end{array}$ & EN33 & $\begin{array}{l}\text { Impactos ambientales negativos significati- } \\
\text { vos, reales y potenciales, en la cadena de } \\
\text { suministro, y medidas al respecto }\end{array}$ & $25 \%$ & $75 \%$ \\
\hline Emisiones & EN20 & $\begin{array}{l}\text { Emisiones de sustancias que agotan el ozo- } \\
\text { no }\end{array}$ & $22 \%$ & $78 \%$ \\
\hline $\begin{array}{l}\text { Productos y Servi- } \\
\text { cios }\end{array}$ & EN28 & $\begin{array}{l}\text { Porcentaje de los productos vendidos y sus } \\
\text { materiales de embalaje que se recuperan al } \\
\text { final de su vida útil }\end{array}$ & $19 \%$ & $81 \%$ \\
\hline
\end{tabular}

Fuente: elaboración propia en base a datos de las MS.

Si se analiza el promedio de presentación de información por aspecto, como se observa en la tabla 4, encabeza la lista Materiales con el 60\%, seguido por Energía $51 \%$ y en menor proporción de presentación los aspectos de Productos y servicios 19\% y Biodiversidad 29\%.

Tabla 4: Porcentaje de presentación promedio por aspecto dentro de la categoría Medio Ambiente.

\begin{tabular}{|l|c|c|}
\hline Aspectos & Reportado & No Reportado \\
\hline Materiales & $60 \%$ & $40 \%$ \\
\hline Energía & $51 \%$ & $49 \%$ \\
\hline General & $50 \%$ & $50 \%$ \\
\hline Efluentes y Residuos & $49 \%$ & $51 \%$ \\
\hline Cumplimiento regulatorio & $47 \%$ & $53 \%$ \\
\hline Agua & $46 \%$ & $54 \%$ \\
\hline
\end{tabular}




\begin{tabular}{|l|c|c|}
\hline $\begin{array}{l}\text { Evaluación ambiental de los } \\
\text { proveedores }\end{array}$ & $42 \%$ & $58 \%$ \\
\hline Reclamación ambiental & $42 \%$ & $58 \%$ \\
\hline Emisiones & $38 \%$ & $62 \%$ \\
\hline Transporte & $33 \%$ & $67 \%$ \\
\hline Biodiversidad & $29 \%$ & $71 \%$ \\
\hline Productos y Servicios & $19 \%$ & $81 \%$ \\
\hline
\end{tabular}

Fuente: elaboración propia en base a datos de las MS

Haciendo el análisis por aspecto, se procedió a recabar información respecto de la forma de presentación de los indicadores, discriminando cinco posibles situaciones:

1) que no estuviese reportado,

2) que estuviese en el informe de sostenibilidad pero no en el índice de contenidos,

3) que la información este en el índice de contenidos y no en la memoria,

4) información en el índice y en la memoria y por último

5) que la información esté en el índice de contenido pero se referencia a un documento distinto a la memoria.

Se obtuvo así la información que figura en la tabla 5.

Tabla 5: Modalidad en la presentación de la información de los indicadores por aspecto.

\begin{tabular}{|c|c|c|c|c|c|c|c|}
\hline Aspecto & & Indicador & $\begin{array}{l}\text { No re- } \\
\text { portado }\end{array}$ & $\begin{array}{c}\text { Se infor- } \\
\text { ma en la } \\
\text { MS pero } \\
\text { no en el } \\
\text { índice } \\
\text { de con- } \\
\text { tenido } \\
\text { (GRI } \\
\text { Index) }\end{array}$ & $\begin{array}{l}\text { Se infor- } \\
\text { ma solo } \\
\text { en el } \\
\text { indice de } \\
\text { conteni- } \\
\text { do }\end{array}$ & $\begin{array}{l}\text { Ref. en el } \\
\text { indice e in- } \\
\text { formación } \\
\text { en la MS }\end{array}$ & $\begin{array}{l}\text { Ref. en } \\
\text { el ind. } \\
\text { de con- } \\
\text { tenido } \\
\text { pero se } \\
\text { refe- } \\
\text { rencia a } \\
\text { un doc. } \\
\text { distinto } \\
\text { a la MS }\end{array}$ \\
\hline \multirow{2}{*}{$\begin{array}{l}\text { Materia- } \\
\text { les }\end{array}$} & EN1 & $\begin{array}{l}\text { Materiales por peso o volu- } \\
\text { men (papel) }\end{array}$ & $31 \%$ & $0 \%$ & $8 \%$ & $61 \%$ & $0 \%$ \\
\hline & EN2 & $\begin{array}{l}\% \text { de los mat. utilizados que } \\
\text { son mat. reciclados (papel) }\end{array}$ & $50 \%$ & $0 \%$ & $14 \%$ & $36 \%$ & $0 \%$ \\
\hline \multirow{5}{*}{ Energía } & EN3 & Consumo energético interno & $19 \%$ & $6 \%$ & $6 \%$ & $69 \%$ & $0 \%$ \\
\hline & EN4 & Consumo energético externo & $72 \%$ & $0 \%$ & $8 \%$ & $20 \%$ & $0 \%$ \\
\hline & EN5 & Intensidad energética & $61 \%$ & $0 \%$ & $3 \%$ & $36 \%$ & $0 \%$ \\
\hline & EN6 & Reducción del consumo energ. & $28 \%$ & $11 \%$ & $8 \%$ & $53 \%$ & $0 \%$ \\
\hline & EN7 & $\begin{array}{l}\text { Reducciones de los requisitos } \\
\text { energ. de los prod. y ss. }\end{array}$ & $64 \%$ & $0 \%$ & $3 \%$ & $33 \%$ & $0 \%$ \\
\hline
\end{tabular}




\begin{tabular}{|c|c|c|c|c|c|c|c|}
\hline \multirow{3}{*}{ Agua } & EN8 & $\begin{array}{l}\text { Captación total de agua s/ } \\
\text { fuente. }\end{array}$ & $42 \%$ & $0 \%$ & $14 \%$ & $44 \%$ & $0 \%$ \\
\hline & EN9 & Fuentes de agua afectadas & $61 \%$ & $3 \%$ & $19 \%$ & $17 \%$ & $0 \%$ \\
\hline & EN10 & $\begin{array}{l}\% \text { y volumen total de agua re- } \\
\text { ciclada y reutilizada. }\end{array}$ & $58 \%$ & $3 \%$ & $11 \%$ & $28 \%$ & $0 \%$ \\
\hline \multirow{4}{*}{$\begin{array}{c}\text { Biodi- } \\
\text { versidad }\end{array}$} & EN11 & $\begin{array}{l}\text { Instalaciones operativas ady., } \\
\text { contengan o estén ubicadas } \\
\text { en áreas protegidas y no pro- } \\
\text { tegidas de gran valor para la } \\
\text { biodiversidad. }\end{array}$ & $67 \%$ & $3 \%$ & $8 \%$ & $22 \%$ & $0 \%$ \\
\hline & EN12 & $\begin{array}{l}\text { Descripción de los impactos } \\
\text { más sig en la biodiversidad. }\end{array}$ & $67 \%$ & $3 \%$ & $6 \%$ & $24 \%$ & $0 \%$ \\
\hline & EN13 & $\begin{array}{l}\text { Hábitats protegidos o restau- } \\
\text { rados }\end{array}$ & $75 \%$ & $3 \%$ & $6 \%$ & $16 \%$ & $0 \%$ \\
\hline & EN14 & $\begin{array}{l}\text { Número de especies cuyos há- } \\
\text { bitats se encuentran en áreas } \\
\text { afectadas por las operaciones. }\end{array}$ & $75 \%$ & $0 \%$ & $6 \%$ & $19 \%$ & $0 \%$ \\
\hline \multirow{7}{*}{$\begin{array}{l}\text { Emisio- } \\
\text { nes }\end{array}$} & EN15 & $\begin{array}{l}\text { Emisiones directas de gases de } \\
\text { efecto invernadero. }\end{array}$ & $42 \%$ & $0 \%$ & $0 \%$ & $58 \%$ & $0 \%$ \\
\hline & EN16 & $\begin{array}{l}\text { Emisiones ind. de GEI o al ge- } \\
\text { nerar energía }\end{array}$ & $56 \%$ & $0 \%$ & $3 \%$ & $41 \%$ & $0 \%$ \\
\hline & EN17 & $\begin{array}{l}\text { Otras emisiones ind. de gases } \\
\text { de efecto invernadero. }\end{array}$ & $72 \%$ & $0 \%$ & $0 \%$ & $28 \%$ & $0 \%$ \\
\hline & EN18 & $\begin{array}{l}\text { Intensidad de las emisiones de } \\
\text { GEI }\end{array}$ & $67 \%$ & $0 \%$ & $0 \%$ & $33 \%$ & $0 \%$ \\
\hline & EN19 & $\begin{array}{l}\text { Reducción de las emisiones de } \\
\text { GEI }\end{array}$ & $56 \%$ & $3 \%$ & $0 \%$ & $42 \%$ & $0 \%$ \\
\hline & EN20 & $\begin{array}{l}\text { Emisiones de sustancias que } \\
\text { agotan el ozono. }\end{array}$ & $78 \%$ & $0 \%$ & $3 \%$ & $19 \%$ & $0 \%$ \\
\hline & EN21 & $\begin{array}{l}\text { Nox, sox y otras emisiones at- } \\
\text { mosféricas significativas. }\end{array}$ & $67 \%$ & $3 \%$ & $3 \%$ & $27 \%$ & $0 \%$ \\
\hline \multirow{6}{*}{$\begin{array}{l}\text { Efluen- } \\
\text { tes y Re- } \\
\text { siduos }\end{array}$} & EN22 & $\begin{array}{l}\text { Vertido total de aguas, según } \\
\text { su calidad y destino. }\end{array}$ & $53 \%$ & $0 \%$ & $8 \%$ & $39 \%$ & $0 \%$ \\
\hline & EN23 & $\begin{array}{l}\text { Peso total de los residuos, s/ } \\
\text { tipo y mét. de tratamiento. }\end{array}$ & $33 \%$ & $6 \%$ & $11 \%$ & $50 \%$ & $0 \%$ \\
\hline & EN24 & $\begin{array}{l}\mathrm{N}^{\circ} \text { y volumen totales de los } \\
\text { derrames significativos. }\end{array}$ & $47 \%$ & $0 \%$ & $28 \%$ & $25 \%$ & $0 \%$ \\
\hline & EN25 & $\begin{array}{l}\text { Residuos transp., importados, } \\
\text { exportados o tratados que se } \\
\text { consideran peligrosos. }\end{array}$ & $58 \%$ & $0 \%$ & $14 \%$ & $28 \%$ & $0 \%$ \\
\hline & EN26 & $\begin{array}{l}\text { Masas de agua y los hábitats } \\
\text { relacionados afectados sig. } \\
\text { por vertidos y efluentes de la } \\
\text { organización. }\end{array}$ & $69 \%$ & $0 \%$ & $17 \%$ & $14 \%$ & $0 \%$ \\
\hline & EN27 & $\begin{array}{l}\text { Mitigación del impacto am- } \\
\text { biental de los prod.y ss. }\end{array}$ & $44 \%$ & $3 \%$ & $3 \%$ & $50 \%$ & $0 \%$ \\
\hline $\begin{array}{l}\text { Produc- } \\
\text { tos y } \\
\text { Servi- } \\
\text { cios }\end{array}$ & EN28 & $\begin{array}{l}\text { \% de los productos vendidos } \\
\text { y sus materiales de embalaje } \\
\text { que se recuperan al final de su } \\
\text { vida útil }\end{array}$ & $81 \%$ & $0 \%$ & $6 \%$ & $13 \%$ & $0 \%$ \\
\hline
\end{tabular}




\begin{tabular}{|c|c|c|c|c|c|c|c|}
\hline $\begin{array}{l}\text { Cumpli- } \\
\text { miento } \\
\text { regula- } \\
\text { torio }\end{array}$ & EN29 & $\begin{array}{l}\text { Valor monetario de las mul- } \\
\text { tas significativas y número de } \\
\text { sanciones no monetarias por } \\
\text { incumplimiento de la legisla- } \\
\text { ción y la normativa ambiental. }\end{array}$ & $53 \%$ & $0 \%$ & $19 \%$ & $25 \%$ & $3 \%$ \\
\hline $\begin{array}{l}\text { Trans- } \\
\text { porte }\end{array}$ & EN30 & $\begin{array}{l}\text { Impactos ambientales signifi- } \\
\text { cativos del transporte de pro- } \\
\text { ductos y otros bienes y mate- } \\
\text { riales }\end{array}$ & $67 \%$ & $0 \%$ & $11 \%$ & $22 \%$ & $0 \%$ \\
\hline General & EN31 & $\begin{array}{l}\text { Desglose de los gastos y las in- } \\
\text { versiones ambientales. }\end{array}$ & $50 \%$ & $3 \%$ & $8 \%$ & $39 \%$ & $0 \%$ \\
\hline \multirow{2}{*}{$\begin{array}{c}\text { Evalua- } \\
\text { ción am- } \\
\text { biental } \\
\text { de los } \\
\text { provee- } \\
\text { dores }\end{array}$} & EN32 & $\begin{array}{l}\text { \% de nuevos proveedores que } \\
\text { se examinaron en función de } \\
\text { criterios ambientales }\end{array}$ & $42 \%$ & $8 \%$ & $11 \%$ & $39 \%$ & $0 \%$ \\
\hline & EN33 & $\begin{array}{l}\text { Impactos amb. negativos sig, } \\
\text { reales y potenciales, en la ca- } \\
\text { dena de suministro, y medi- } \\
\text { das al respecto. }\end{array}$ & $75 \%$ & $3 \%$ & $11 \%$ & $11 \%$ & $0 \%$ \\
\hline $\begin{array}{l}\text { Recla- } \\
\text { mación } \\
\text { ambien- } \\
\text { tal }\end{array}$ & EN34 & $\begin{array}{l}\mathrm{N}^{\mathrm{o}} \text { de reclamaciones ambien- } \\
\text { tales que se han presentado, } \\
\text { abordado y resuelto mediante } \\
\text { mecanismos formales de re- } \\
\text { clamación. }\end{array}$ & $58 \%$ & $0 \%$ & $28 \%$ & $11 \%$ & $3 \%$ \\
\hline
\end{tabular}

Fuente: elaboración propia en base a datos de las MS.

Se observa que en la mayoría de los indicadores presentados la información se encuentra en gran porcentaje en el cuerpo de la memoria y en el índice de contenido. Indicadores como número y volúmenes totales de los derrames significativos (EN24), Hábitats afectados por efluentes y residuos (EN26), fuentes de agua afectadas (EN9) y reclamaciones ambientales (EN34) se reportan en mayor porcentaje solamente en el índice de contenido.

\section{Nuevos Estándares GRI}

A partir del mes de julio del 2018, las organizaciones que quieran seguir los lineamientos del GRI para realizar sus reportes de sustentabilidad deberán aplicar los nuevos estándares elaborados por la Global Reporting Initiative. La novedad que incorporan estos estándares, publicados a finales del año 2016, respecto de la guía G4 es que presenta una estructura basada en módulos interrelacionados permitiendo mayor flexibilidad en la actualización de los temas.

Se establecieron de esta forma 36 estándares agrupados en: tres estándares universales denominados (Fundamentos, Contenidos Generales y Enfoque de Gestión) y tres específicos sobre temas económicos, ambientales y sociales.

De los 33 indicadores que presenta la guía G4 para el aspecto medio ambiente, se realiza una adaptación de los mismos en los nuevos estándares, quedando así configurados dentro del estándar ambiental (serie 300) 8 estándares temáticos (materiales, energía, agua, biodiversidad, emisiones, efluentes y residuos, cumplimiento ambiental y evaluación ambiental de los provee- 
dores) que informan sobre los impactos materiales de una organización en lo que respecta a los temas ambientales.

En los nuevos estándares se reagrupan en otros ejes temáticos los aspectos de: productos y servicios, transporte, general, mecanismos de reclamación en materia ambiental que estaban contemplados en la guía G4.

A continuación se pueden observar en las tablas 6 a 8 el análisis entre la última guía GRI y los nuevos estándares en lo que respecta a medio ambiente.

Tabla 6: Indicadores que no presentan cambios entre G4 y estándares GRI.

\begin{tabular}{|c|c|c|c|}
\hline $\begin{array}{l}\text { Ejes } \\
\text { Temáticos }\end{array}$ & Guía G4 & $\begin{array}{l}\text { Estándares } \\
\text { temáticos }\end{array}$ & Denominación \\
\hline Materiales & EN1 & $301-1$ & Materiales usados por peso y volumen \\
\hline Energía & EN7 & $302-5$ & Reducciones en los requisitos de energía de productos y servicios \\
\hline Agua & EN8 & $303-1$ & Extracción de agua por fuente \\
\hline \multirow{4}{*}{$\begin{array}{l}\text { Biodiversi- } \\
\text { dad }\end{array}$} & EN11 & $304-1$ & $\begin{array}{l}\text { Sitios operacionales de propiedad, rentados, administrados o ad- } \\
\text { yacentes a áreas protegidas y áreas de alto valor de biodiversidad } \\
\text { fuera de las áreas protegidas }\end{array}$ \\
\hline & EN12 & $304-2$ & $\begin{array}{l}\text { Impactos significativos de actividades, productos y servicios en la } \\
\text { biodiversidad }\end{array}$ \\
\hline & EN13 & $304-3$ & Hábitats protegidos o restaurados \\
\hline & EN14 & $304-4$ & $\begin{array}{l}\text { Especies de la Lista Roja de la UICN y especies de la lista nacional } \\
\text { de conservación con hábitats en áreas afectadas por las operacio- } \\
\text { nes }\end{array}$ \\
\hline $\begin{array}{l}\text { Efluentes y } \\
\text { Residuos }\end{array}$ & EN24 & $306-3$ & Derrames significativos \\
\hline \multirow{2}{*}{$\begin{array}{l}\text { Evaluación } \\
\text { ambiental } \\
\text { de los pro- } \\
\text { veedores }\end{array}$} & EN32 & $308-1$ & $\begin{array}{l}\text { Nuevos proveedores que fueron seleccionados utilizando criterios } \\
\text { ambientales }\end{array}$ \\
\hline & EN33 & $308-2$ & $\begin{array}{l}\text { Impactos ambientales negativos en la cadena de suministro y ac- } \\
\text { ciones tomadas }\end{array}$ \\
\hline
\end{tabular}

Fuente: elaboración propia en base a datos de globalreporting.org

Tabla 7: Indicadores de la Guía G4 eliminados de los estándares para reducir la duplicación de información.

\begin{tabular}{|l|c|l|}
\hline \multicolumn{1}{|c|}{ Ejes Temáticos } & Guía G4 & \multicolumn{1}{c|}{ Denominación } \\
\hline Productos y Servicios & EN27 & $\begin{array}{l}\text { Grado de mitigación del impacto ambiental de los productos y } \\
\text { servicios }\end{array}$ \\
\hline Transporte & EN30 & $\begin{array}{l}\text { Impactos ambientales significativos del transporte de productos } \\
\text { y otros bienes y materiales utilizados para las actividades de la } \\
\text { organización, así como del transporte de personal }\end{array}$ \\
\hline Aspecto General & EN31 & Desglose de los gastos y las inversiones ambientales \\
\hline
\end{tabular}

Fuente: elaboración propia en base a datos de globalreporting.org 
Tabla 8: Indicadores de la Guía G4 a los que se les incorpora información y / o modificaciones en los nuevos estándares temáticos.

\begin{tabular}{|c|c|c|c|c|}
\hline $\begin{array}{l}\text { Ejes } \\
\text { Temáticos }\end{array}$ & Guía G4 & $\begin{array}{l}\text { Estánda- } \\
\text { res temá- } \\
\text { ticos }\end{array}$ & Denominación & Nuevas consideraciones \\
\hline \multirow[b]{2}{*}{ Materiales } & EN2 & $301-2$ & $\begin{array}{l}\text { Materiales de entrada } \\
\text { reciclados utilizados }\end{array}$ & $\begin{array}{l}\text { Cálculos en la presentación de la in- } \\
\text { formación }\end{array}$ \\
\hline & EN28 & $301-3$ & $\begin{array}{l}\text { Productos recuperados } \\
\text { y sus materiales de em- } \\
\text { balaje }\end{array}$ & $\begin{array}{l}\text { Cálculos en la presentación de la in- } \\
\text { formación }\end{array}$ \\
\hline \multirow{4}{*}{ Energía } & EN3 & $302-1$ & $\begin{array}{l}\text { Consumo de energía den- } \\
\text { tro de la organización }\end{array}$ & $\begin{array}{l}\text { Cálculos en la presentación de la infor- } \\
\text { mación y evitar duplicaciones }\end{array}$ \\
\hline & EN4 & $302-2$ & $\begin{array}{l}\text { Consumo de energía fuera } \\
\text { de la organización }\end{array}$ & $\begin{array}{l}\text { Descripción del enfoque e información } \\
\text { adicional }\end{array}$ \\
\hline & EN5 & $302-3$ & Intensidad energética & $\begin{array}{l}\text { Cálculos en la presentación de la in- } \\
\text { formación }\end{array}$ \\
\hline & EN6 & $302-4$ & $\begin{array}{l}\text { Reducción del consumo } \\
\text { de energía }\end{array}$ & $\begin{array}{l}\text { Exclusión de información, descripción } \\
\text { de mediciones y métodos empleados }\end{array}$ \\
\hline \multirow[t]{2}{*}{ Agua } & EN9 & $303-2$ & $\begin{array}{l}\text { Fuentes de agua significa- } \\
\text { tivamente afectadas por la } \\
\text { extracción de agua }\end{array}$ & $\begin{array}{l}\text { Cálculos en la presentación de la in- } \\
\text { formación }\end{array}$ \\
\hline & EN10 & $303-3$ & $\begin{array}{l}\text { Agua reciclada y reutili- } \\
\text { zada }\end{array}$ & Se adiciona información \\
\hline \multirow{7}{*}{ Emisiones } & EN15 & $305-1$ & Emisiones de GEI Directas & $\begin{array}{l}\text { Cálculos en la presentación de la infor- } \\
\text { mación y exclusión de información }\end{array}$ \\
\hline & EN16 & $305-2$ & Energía indirecta & $\begin{array}{l}\text { Cálculos en la presentación de la infor- } \\
\text { mación y exclusión de información }\end{array}$ \\
\hline & EN17 & $305-3$ & Otras emisiones indirectas & $\begin{array}{l}\text { Cálculos en la presentación de la infor- } \\
\text { mación y exclusión de información }\end{array}$ \\
\hline & EN18 & $305-4$ & $\begin{array}{l}\text { Intensidad de las emisio- } \\
\text { nes de GEI }\end{array}$ & $\begin{array}{l}\text { Cálculos en la presentación de la in- } \\
\text { formación }\end{array}$ \\
\hline & EN19 & $305-5$ & $\begin{array}{l}\text { Reducción de emisiones } \\
\text { de GEI }\end{array}$ & $\begin{array}{l}\text { Cálculos en la presentación de la infor- } \\
\text { mación y exclusión de información }\end{array}$ \\
\hline & EN20 & $305-6$ & $\begin{array}{l}\text { Emisiones de sustancias } \\
\text { que agotan la capa de ozo- } \\
\text { no (SAO) }\end{array}$ & $\begin{array}{l}\text { Cálculos en la presentación de la in- } \\
\text { formación }\end{array}$ \\
\hline & EN21 & $305-7$ & $\begin{array}{l}\text { NOX, SOX y otras emisio- } \\
\text { nes atmosféricas signifi- } \\
\text { cativas }\end{array}$ & $\begin{array}{l}\text { Cálculos en la presentación de la in- } \\
\text { formación }\end{array}$ \\
\hline \multirow{4}{*}{$\begin{array}{l}\text { Efluentes y } \\
\text { residuos }\end{array}$} & EN22 & $306-1$ & $\begin{array}{l}\text { Descarga de agua por } \\
\text { calidad y destino }\end{array}$ & $\begin{array}{l}\text { Cálculos en la presentación de la in- } \\
\text { formación }\end{array}$ \\
\hline & EN23 & $306-2$ & $\begin{array}{l}\text { Residuos por tipo y méto- } \\
\text { do de eliminación }\end{array}$ & $\begin{array}{l}\text { Cálculos en la presentación de la in- } \\
\text { formación }\end{array}$ \\
\hline & EN25 & $306-4$ & $\begin{array}{l}\text { Transporte de residuos } \\
\text { peligrosos }\end{array}$ & $\begin{array}{l}\text { Cálculos en la presentación de la infor- } \\
\text { mación y descripción metodología }\end{array}$ \\
\hline & EN26 & $306-5$ & $\begin{array}{l}\text { Cuerpos de agua afecta- } \\
\text { dos por descargas de agua } \\
\text { y / o escorrentía }\end{array}$ & $\begin{array}{l}\text { Cálculos en la presentación de la in- } \\
\text { formación }\end{array}$ \\
\hline $\begin{array}{l}\text { Cumpli- } \\
\text { miento am- } \\
\text { biental }\end{array}$ & EN29 & $307-1$ & $\begin{array}{l}\text { Incumplimiento de la } \\
\text { legislación y normativa } \\
\text { ambiental }\end{array}$ & $\begin{array}{l}\text { Se aclara que las organizaciones deben } \\
\text { informar sobre los incumplimientos } \\
\text { de las leyes y normas. }\end{array}$ \\
\hline
\end{tabular}

Fuente: elaboración propia en base a datos de globalreporting.org 
De las organizaciones de la muestra, la primera que realizó la implementación de los estándares del GRI 2016 para su informe de sustentabilidad es el Banco Galicia para su reporte anual del 2016. Teniendo en cuenta que a la fecha de corte de esta investigación también se encuentra presentado el informe anual 2017 (continuando con la aplicación de los estándares), se procedió a realizar un análisis sobre la información referida al aspecto Medio Ambiente comparando los reportes de esta entidad del año 2015 (bajo guía G4), 2016 y 2017 (estos últimos bajo estándar GRI). Se pretende observar similitudes, diferencias, modificación e incorporación de información bajo la nueva herramienta que luego todas las organizaciones deberían aplicar.

Tabla 9: Cuadro comparativo sobre información contenida en las MS del Banco Galicia respecto a Medio Ambiente para los períodos 2015, 2016 y 2017.

\begin{tabular}{|c|c|c|c|c|c|c|}
\hline $\begin{array}{c}\text { Ejes } \\
\text { Temáticos }\end{array}$ & $\begin{array}{c}\text { Guía } \\
\text { G4 }\end{array}$ & $\begin{array}{l}\text { Denomi- } \\
\text { nación }\end{array}$ & $\begin{array}{c}2015 \\
\text { (G4 - GRI) }\end{array}$ & $\begin{array}{l}\text { Estándares } \\
\text { temáticos }\end{array}$ & $\begin{array}{l}2016 \\
\text { (Estándar - GRI) }\end{array}$ & $\begin{array}{l}2017 \\
\text { (Estándar-GRI) }\end{array}$ \\
\hline Materiales & EN1 & $\begin{array}{l}\text { Materiales } \\
\text { usados } \\
\text { por peso y } \\
\text { volumen }\end{array}$ & $\begin{array}{l}\text { Informa TN } \\
\text { de consumo } \\
\text { de papel }\end{array}$ & $301-1$ & $\begin{array}{l}\text { Informa TN de } \\
\text { consumo de } \\
\text { papel }\end{array}$ & $\begin{array}{l}\text { Informa TN de } \\
\text { consumo de } \\
\text { papel }\end{array}$ \\
\hline Energía & EN7 & $\begin{array}{l}\text { Reduccio- } \\
\text { nes en los } \\
\text { requisitos } \\
\text { de energía } \\
\text { de pro- } \\
\text { ductos y } \\
\text { servicios }\end{array}$ & $\begin{array}{l}\text { Acciones para } \\
\text { reducir el con- } \\
\text { sumo energéti- } \\
\text { co (luminaria, } \\
\text { ascensores, } \\
\text { calefacción, } \\
\text { etc) }\end{array}$ & $302-5$ & $\begin{array}{l}\text { Acciones para } \\
\text { reducir el con- } \\
\text { sumo energético } \\
\text { (luminaria, aires } \\
\text { acondicionados, } \\
\text { cartelería, etc) }\end{array}$ & $\begin{array}{l}\text { Acciones para } \\
\text { reducir el cons. } \\
\text { energético (lu- } \\
\text { minaria, aires } \\
\text { acond., prototipo } \\
\text { de suc. domótica, } \\
\text { etc) }\end{array}$ \\
\hline Agua & EN8 & $\begin{array}{l}\text { Extracción } \\
\text { de agua } \\
\text { por fuente }\end{array}$ & $\begin{array}{l}\text { Consumo en } \\
\text { m3 }\end{array}$ & $303-1$ & Consumo en m3 & No se informa \\
\hline \multirow{4}{*}{$\begin{array}{l}\text { Biodiversi- } \\
\text { dad }\end{array}$} & EN11 & $\begin{array}{l}\text { Sitios } \\
\text { operacio- } \\
\text { nales ady. } \\
\text { a áreas } \\
\text { protegidas } \\
\text { y áreas de } \\
\text { alto valor } \\
\text { de biodi- } \\
\text { versidad. }\end{array}$ & No se informa & $304-1$ & No se informa & No se informa \\
\hline & EN12 & $\begin{array}{l}\text { Impactos } \\
\text { signif. } \\
\text { de acti- } \\
\text { vidades, } \\
\text { prod.y ss. } \\
\text { en la bio- } \\
\text { diversidad } \\
\end{array}$ & No se informa & $304-2$ & No se informa & No se informa \\
\hline & EN13 & $\begin{array}{l}\text { Hábitats } \\
\text { protegidos } \\
\text { o restaura- } \\
\text { dos }\end{array}$ & No se informa & $304-3$ & No se informa & No se informa \\
\hline & EN14 & $\begin{array}{l}\text { Especies } \\
\text { con há- } \\
\text { bitats en } \\
\text { áreas afec- } \\
\text { tadas por } \\
\text { las op. }\end{array}$ & No se informa & $304-4$ & No se informa & No se informa \\
\hline
\end{tabular}




\begin{tabular}{|c|c|c|c|c|c|c|}
\hline $\begin{array}{l}\text { Efluentes y } \\
\text { Residuos }\end{array}$ & EN24 & $\begin{array}{l}\text { Derrames } \\
\text { significati- } \\
\text { vos }\end{array}$ & $\begin{array}{l}\text { No se produ- } \\
\text { jeron derra- } \\
\text { mes. }\end{array}$ & $306-3$ & $\begin{array}{l}\text { No se produje- } \\
\text { ron derrames. }\end{array}$ & $\begin{array}{l}\text { No se produjeron } \\
\text { derrames. }\end{array}$ \\
\hline \multirow{2}{*}{$\begin{array}{l}\text { Evaluación } \\
\text { ambiental de } \\
\text { los provee- } \\
\text { dores }\end{array}$} & EN32 & $\begin{array}{l}\text { Nuevos } \\
\text { proveedo- } \\
\text { res selec- } \\
\text { cionados } \\
\text { utilizando } \\
\text { criterios } \\
\text { ambien- } \\
\text { tales }\end{array}$ & $\begin{array}{l}\text { Acciones reali- } \\
\text { zadas }\end{array}$ & $308-1$ & No se informa & $\begin{array}{l}\text { Acciones reali- } \\
\text { zadas }\end{array}$ \\
\hline & EN33 & $\begin{array}{l}\text { Imp. am- } \\
\text { bientales } \\
\text { negativos } \\
\text { en la ca- } \\
\text { dena de } \\
\text { suministro } \\
\text { y acciones } \\
\text { tomadas }\end{array}$ & No se informa & $308-2$ & $\begin{array}{l}\text { No se detectaron } \\
\text { impactos am- } \\
\text { bientales neg. }\end{array}$ & $\begin{array}{l}\text { No se detectaron } \\
\text { impactos ambien- } \\
\text { tales neg. }\end{array}$ \\
\hline \multicolumn{7}{|c|}{ Indicadores que presentan modificaciones en los nuevos estándares respecto de la guía G4 } \\
\hline \multirow[t]{2}{*}{ Materiales } & EN2 & $\begin{array}{l}\text { Materiales } \\
\text { de entrada } \\
\text { reciclados } \\
\text { utilizados }\end{array}$ & $\begin{array}{l}\text { Informa TN } \\
\text { de papel re- } \\
\text { ciclado, cant. } \\
\text { bolsas de } \\
\text { polietileno } \\
\text { oxiobiod. }\end{array}$ & $301-2$ & $\begin{array}{l}\text { Informa TN de } \\
\text { papel reciclado, } \\
\text { cant.bolsas de } \\
\text { polietileno ox., } \\
\text { sobres reutili- } \\
\text { zables }\end{array}$ & $\begin{array}{l}\text { Informa TN de } \\
\text { papel reciclado, } \\
\text { cant.bolsas de } \\
\text { polietileno ox., so- } \\
\text { bres reutilizables }\end{array}$ \\
\hline & EN28 & $\begin{array}{l}\text { Produ. rec } \\
\text { y sus em- } \\
\text { balajes }\end{array}$ & $\begin{array}{l}\text { Inf. que no } \\
\text { recup. }\end{array}$ & $301-3$ & No se informa & No se informa \\
\hline \multirow{4}{*}{ Energía } & EN3 & $\begin{array}{l}\text { Consumo } \\
\text { de energía } \\
\text { dentro de } \\
\text { la organi- } \\
\text { zación }\end{array}$ & $\begin{array}{l}\text { Consumo de } \\
\text { gas, electrici- } \\
\text { dad, gasoil y } \\
\text { nafta }\end{array}$ & $302-1$ & $\begin{array}{l}\text { Consumo de } \\
\text { gas, electrici- } \\
\text { dad, gasoil y } \\
\text { nafta }\end{array}$ & $\begin{array}{l}\text { Consumo de gas, } \\
\text { electricidad, gasoil } \\
\text { y nafta }\end{array}$ \\
\hline & EN4 & $\begin{array}{l}\text { Consumo } \\
\text { de energía } \\
\text { fuera de la } \\
\text { organiza- } \\
\text { ción }\end{array}$ & $\begin{array}{l}\text { No se infor- } \\
\text { ma }\end{array}$ & $302-2$ & $\begin{array}{l}\text { Informa que no } \\
\text { se dispone de } \\
\text { este dato }\end{array}$ & $\begin{array}{l}\text { Informa que no } \\
\text { se dispone de este } \\
\text { dato }\end{array}$ \\
\hline & EN5 & $\begin{array}{l}\text { Intensidad } \\
\text { energética }\end{array}$ & $\begin{array}{l}\mathrm{Kw} / \mathrm{m} 3 / \text { año } \\
\text { sucursales y } \\
\text { edificio cen- } \\
\text { tral }\end{array}$ & $302-3$ & $\begin{array}{l}\mathrm{Kw} / \mathrm{m} 3 / \text { año } \\
\text { sucursales y } \\
\text { edificio central }\end{array}$ & $\begin{array}{l}\mathrm{Kw} / \mathrm{m} 3 / \text { año su- } \\
\text { cursales y edificio } \\
\text { central }\end{array}$ \\
\hline & EN6 & $\begin{array}{l}\text { Reducción } \\
\text { del con- } \\
\text { sumo de } \\
\text { energía }\end{array}$ & $\begin{array}{l}\text { \% de re- } \\
\text { ducción de } \\
\text { energía edif. } \\
\text { central y su- } \\
\text { cursales }\end{array}$ & $302-4$ & $\begin{array}{l}\text { \% de reducción } \\
\text { de energía edif. } \\
\text { central y sucur- } \\
\text { sales }\end{array}$ & $\begin{array}{l}\text { \% de reducción de } \\
\text { energía edif.cen- } \\
\text { tral y sucursales }\end{array}$ \\
\hline
\end{tabular}




\begin{tabular}{|c|c|c|c|c|c|c|}
\hline \multirow[t]{2}{*}{ Agua } & EN9 & $\begin{array}{l}\text { Fuentes } \\
\text { de agua } \\
\text { significa- } \\
\text { tivamente } \\
\text { afectadas } \\
\text { por la } \\
\text { extracción } \\
\text { de agua }\end{array}$ & $\begin{array}{l}\text { No se infor- } \\
\text { ma }\end{array}$ & $303-2$ & $\begin{array}{l}\text { Procedencia } \\
\text { del recurso (red } \\
\text { pública y per- } \\
\text { foración) }\end{array}$ & No se informa \\
\hline & EN10 & $\begin{array}{l}\text { Agua re- } \\
\text { ciclada y } \\
\text { reutilizada }\end{array}$ & $\begin{array}{l}\text { No se infor- } \\
\text { ma }\end{array}$ & $303-3$ & $\begin{array}{l}\text { Se informa del } \\
\text { reciclaje de } \\
\text { agua de lluvia } \\
\text { de una suc. }\end{array}$ & No se informa \\
\hline \multirow{7}{*}{ Emisiones } & EN15 & $\begin{array}{l}\text { Emisiones } \\
\text { directas } \\
\text { de gases } \\
\text { de efecto } \\
\text { inverna- } \\
\text { dero }\end{array}$ & $\begin{array}{l}\text { Informa TN } \\
\text { de CO3 equi- } \\
\text { valente }\end{array}$ & $305-1$ & $\begin{array}{l}\text { Informa } \mathrm{TN} \text { de } \\
\mathrm{CO} 3 \text { equiva- } \\
\text { lente }\end{array}$ & $\begin{array}{l}\text { Informa TN de } \\
\text { CO3 equivalente }\end{array}$ \\
\hline & EN16 & $\begin{array}{l}\text { Emisiones } \\
\text { indirectas } \\
\text { de gases } \\
\text { de efecto } \\
\text { inverna- } \\
\text { dero }\end{array}$ & $\begin{array}{l}\text { Informa TN } \\
\text { de CO3 equi- } \\
\text { valente }\end{array}$ & $305-2$ & $\begin{array}{l}\text { Informa } \mathrm{TN} \text { de } \\
\text { CO3 equiva- } \\
\text { lente }\end{array}$ & $\begin{array}{l}\text { Informa TN de } \\
\text { CO3 equivalente }\end{array}$ \\
\hline & EN17 & $\begin{array}{l}\text { Otras } \\
\text { emisiones } \\
\text { indirectas }\end{array}$ & $\begin{array}{l}\text { Informa TN } \\
\text { de CO3 equi- } \\
\text { valente }\end{array}$ & $305-3$ & $\begin{array}{l}\text { Informa TN de } \\
\text { CO3 equiva- } \\
\text { lente }\end{array}$ & $\begin{array}{l}\text { Informa TN de } \\
\text { CO3 equivalente }\end{array}$ \\
\hline & EN18 & $\begin{array}{l}\text { Int. de las } \\
\text { emisiones } \\
\text { de GEI }\end{array}$ & $\begin{array}{l}\text { No se infor- } \\
\text { ma }\end{array}$ & $305-4$ & No se informa & No se informa \\
\hline & EN19 & $\begin{array}{l}\text { Reducción } \\
\text { de emisio- } \\
\text { nes GEI }\end{array}$ & $\begin{array}{l}\text { No se infor- } \\
\text { ma }\end{array}$ & $305-5$ & No se informa & No se informa \\
\hline & EN20 & $\begin{array}{l}\text { Emis. que } \\
\text { agotan el } \\
\text { ozono }\end{array}$ & $\begin{array}{l}\text { No se infor- } \\
\text { ma }\end{array}$ & $305-6$ & No se informa & No se informa \\
\hline & EN21 & $\begin{array}{l}\text { NOX, SOX } \\
\text { y otras } \\
\text { emisiones } \\
\text { atmosféri- } \\
\text { cas sig. }\end{array}$ & $\begin{array}{l}\text { Informa que } \\
\text { no aplica el } \\
\text { indicador }\end{array}$ & $305-7$ & $\begin{array}{l}\text { Informa que no } \\
\text { aplica el indi- } \\
\text { cador }\end{array}$ & No se informa \\
\hline
\end{tabular}




\begin{tabular}{|c|c|c|c|c|c|c|}
\hline \multirow{4}{*}{$\begin{array}{l}\text { Efluentes y } \\
\text { residuos }\end{array}$} & EN22 & $\begin{array}{l}\text { Descarga } \\
\text { de agua } \\
\text { por ca- } \\
\text { lidad y } \\
\text { destino }\end{array}$ & $\begin{array}{l}\text { Serv.sanit.pu- } \\
\text { blico y pozos } \\
\text { absorbentes. }\end{array}$ & $306-1$ & $\begin{array}{l}\text { Serv.sanit.pu- } \\
\text { blico y pozos } \\
\text { absorbentes. }\end{array}$ & No se informa \\
\hline & EN23 & $\begin{array}{l}\text { Residuos } \\
\text { por tipo y } \\
\text { método de } \\
\text { elimina- } \\
\text { ción }\end{array}$ & $\begin{array}{l}\text { En las suc. } \\
\text { no se realizan } \\
\text { mediciones } \\
\text { aunque sí se } \\
\text { gestionan los } \\
\text { residuos. }\end{array}$ & $306-2$ & $\begin{array}{l}\text { En las sucursa- } \\
\text { les, no se reali- } \\
\text { zan mediciones } \\
\text { aunque sí se } \\
\text { gestionan los } \\
\text { residuos. }\end{array}$ & $\begin{array}{l}\text { Se informa sobre } \\
\text { residuos especia- } \\
\text { les. }\end{array}$ \\
\hline & EN25 & $\begin{array}{l}\text { Transporte } \\
\text { de resi- } \\
\text { duos peli- } \\
\text { grosos }\end{array}$ & $\begin{array}{l}\text { Los residuos } \\
\text { peligrosos } \\
\text { son transp. en } \\
\text { forma local. }\end{array}$ & $306-4$ & $\begin{array}{l}\text { Los residuos } \\
\text { peligrosos son } \\
\text { transp. en for- } \\
\text { ma local. }\end{array}$ & No se informa \\
\hline & EN26 & $\begin{array}{l}\text { Cuerpos } \\
\text { de agua } \\
\text { afectados } \\
\text { por des- } \\
\text { cargas de } \\
\text { agua y efl. }\end{array}$ & $\begin{array}{l}\text { No se infor- } \\
\text { ma }\end{array}$ & $306-5$ & No se informa & No se informa \\
\hline $\begin{array}{l}\text { Cumplimien- } \\
\text { to ambiental }\end{array}$ & EN29 & $\begin{array}{l}\text { Incumpli- } \\
\text { miento de } \\
\text { la legisla- } \\
\text { ción y n. } \\
\text { ambiental }\end{array}$ & Sin sanciones & $307-1$ & Sin sanciones & Sin sanciones \\
\hline \multicolumn{7}{|c|}{ Indicadores suprimidos del Estándar Medio Ambiente } \\
\hline $\begin{array}{l}\text { Productos y } \\
\text { Servicios }\end{array}$ & EN27 & $\begin{array}{l}\text { Grado de } \\
\text { mitiga- } \\
\text { ción del } \\
\text { impacto } \\
\text { ambiental } \\
\text { de los } \\
\text { productos } \\
\text { y servi- } \\
\text { cios }\end{array}$ & \multicolumn{4}{|c|}{ Transformación de las sucursales en Eco-Responsables } \\
\hline Transporte & EN30 & $\begin{array}{l}\text { Imp. } \\
\text { Amb. } \\
\text { significa- } \\
\text { tivos del } \\
\text { transpor- } \\
\text { te de prod } \\
\text { bs y mat. } \\
\text { utilizados }\end{array}$ & \multicolumn{4}{|l|}{ No se informa } \\
\hline $\begin{array}{l}\text { Aspecto Ge- } \\
\text { neral }\end{array}$ & EN31 & $\begin{array}{l}\text { Desglose } \\
\text { de los } \\
\text { gastos y } \\
\text { las inver- } \\
\text { siones } \\
\text { ambien- } \\
\text { tales }\end{array}$ & \multicolumn{4}{|l|}{ No se informa } \\
\hline
\end{tabular}

Fuente: elaboración propia en base a datos de las MS 
Como se puede visualizar en el análisis comparativo de la tabla 9, esta organización no presenta grandes cambios significativos en la exposición de la información sobre la temática de Medio Ambiente, teniendo en cuenta tanto la aplicación de la guía G4 como de los estándares GRI.

\section{CONCLUSIONES}

Actualmente la incompleta utilidad de la información financiera y la notable evolución de la información no financiera nos desafía a presentar informes de rendición de cuentas que sirvan no sólo a inversores y acreedores sino a todos los públicos de interés. El avance de estos informes exige como otrora sucedió con los informes financieros, que exista homogeneidad entre los mismos para que quienes los analicen puedan comparar y entender con claridad que es lo que se está informando.

Motivados por esta realidad varias organizaciones han propuesto diversos modelos o guías buscando estandarizar la presentación de la información no financiera, logrando desde hace unos años acaparar este espacio la guía del Global Reporting Initiative.

En el presente trabajo se propuso, ante la falta de normas que estandaricen la información no financiera, realizar un análisis de contenido de los aspectos ambientales de los reportes de sostenibilidad presentados por las empresas cotizantes de argentina. El resultado de este análisis como se evidencia en el desarrollo de esta investigación y teniendo en cuenta que las empresas analizadas están trabajando con las mismas guías de presentación, deja plasmada la falta de homogenización de la información que se presenta, aspecto clave y relevante al momento de presentar información a terceros, lo cual lleva a preguntar si realmente son eficientes estas guías para la presentación de información no financiera o deberíamos pensar en guías con parámetros más exigentes para el reporte de las organizaciones?

A partir del mes de Julio del 2018 la GRI propone mutar de las guías GRI G4 a los nuevos "GRI Standards" entendiéndose como una instancia superadora. Frente a este nuevo panorama se esperaba que estos estándares lograran superar las deficiencias de las anteriores guías. Lamentablemente no logran mejorar o sortear las dificultades de uniformar la información presentada por las organizaciones. Tal como se demuestra en la última parte del trabajo donde se analiza el caso del Banco Galicia, la información presentada y la forma que la misma se expone no exterioriza casi ningún cambio entre el reporte 2015 utilizando la Guía GRI G4 y los posteriores reportes 2016 y 2017 donde se implementan los GRI Standards. Estamos frente a cambios de modelos que no generan modificaciones sustanciales en la forma de reportar de las organizaciones, ni en la calidad de la información que presenta.

Es un gran desafío el que tenemos que asumir como profesionales de las ciencias económicas el de reportar información financiera pero también social y ambiental. Son numerosos los estudios que desde hace varios años se vienen presentando sobre la importancia y utilidad de la información no financiera, es hora que la profesión en ciencias económicas asuma este rol tan importante que la sociedad en general le está demandando y nos transformemos en profesionales que generen valor con su informes a la sociedad. 


\section{REFERENCIAS BIBLIOGRÁFICAS}

Alonso-Almeida, M., Marimon, F. and Llach, J. (2015). Difusión de las memorias de sostenibilidad en Latinoamérica: análisis territorial y sectorial. Estudios Gerenciales. 31(135), 139-149.

Bellantuono, N., Pontrandolfo, P., \& Scozzi, B. (2016). Capturing the stakeholders' view in sustainability reporting: a novel approach. Sustainability. 8(4), 379.

Ernst \& Young SL (2017). Is your nonfinancial performance revealing the true value of your business to investors?. Disponible en: https: / / www.ey.com/Publication/vwLUAssets / EY_-_ Nonfinancial_performance_may_influence_investors/\$FILE/ey-nonfinancial-performance-may-influence-investors.pdf

Garcia Fronti, I. (2012). Problemas de la Contabilidad social y ambiental: Algunas reflexiones sobre la necesidad de un abordaje interdisciplinario. Contaduría Universidad de Antioquia, (60), 209-218.

Global Reporting Initiative (GRI) (2013). G4 Sustainability Reporting Guidelines. Reporting Principles and Standard Disclosures, Global Reporting Initiative, Amsterdam.

Global Reporting Initiative (GRI) (2016). GRI Standards. Disponible en: https: / / www.globalreporting.org/standards

Gray, R., Kouhy, R. y Lavers, S. (1995). Corporate social and environmental reporting - a review of the literature and longitudinal study of UK disclosure. Accounting, Auditing $\mathcal{E}$ Accountability Journal. 8 (2), 47-77.

Guthrie, J., Petty, R., Yongvanich, K. y Ricceri, F. (2004). Using content analysis as a research method to inquire into intellectual capital reporting. Journal of Intellectual Capital. 5(2), 282-293.

Hauque, S. M., Di Russo de Hauque L., del Barco J. P. y del Barco M. A. (2016). Responsabilidad Social Empresarial: una mirada axiológica. Santa Fe, Argentina: Ediciones UNL.

Jara-Sarrúa, L. (2017). Comparabilidad de las Memorias de Sostenibilidad elaboradas con el estándar GRI-G4 opción Core. El caso de Argentina, Chile, Colombia, México y Perú. Presented at 11th Centre for Social and Environmental Accounting Research (CSEAR) Spain Conference. Zaragoza, España.

Kerste M., Rosenboom N., Sikken B.J. y Weda J. (2011). Financing Sustainability - Insights for Investors, Corporate Executives and Policymakers. Amsterdam, Holanda: VU University Press.

Krippendorff, K. (1990). Metodología de análisis de contenido. Teoría y Práctica. Barcelona, España: Piados Comunicación.

Larrinaga, C., Moneva, J. M., Llena, F., Carrasco, F., \& Correa, C. (2002). Regulación contable de la información medioambiental. Normativa española e internacional. Aeca, Madrid.

Moneva, J. M. (2005a). Información sobre responsabilidad social corporativa: Situación y tendencias. RAE: Revista Asturiana de Economía, (34), 43-67.

Moneva, J. M. (2005b). La verificación externa de los informes de sostenibilidad: el aseguramiento. Disponible en: https:/ / ecodes.org/noticias/la-verificaci\%C3\%B3n-externa-de-los-informes-de-sostenibilidad-el-aseguramiento\#.W0fp3TpKjIU

Pahlen Acuña, R., Fronti de Garcia, L.(2004) Contabilidad social y ambiental. Buenos Aires: Ediciones Macchi.

Tejedo Romero, F. (2016). Información de los recursos intangibles ocultos: ¿memorias de sostenibilidad o informe anual?. European Research on Management and Business Economics. 22(2), 101-109.

Zenghelis, D., \& Stern, N. (2016). The importance of looking forward to manage risks: submission to the Task Force on Climate-Related Financial Disclosures. Disponible en: http:/ / www. lse.ac.uk / GranthamInstitute / publication / the-importance-of-looking-forward-to-manage-risks-submission-to-the-task-force-on-climate-related-financial-disclosures / 